\title{
Oscillation criteria for certain third-order delay dynamic equations
}

Jiashan Yang*

\author{
"Correspondence: syxyyjs@163.com \\ Department of Science and \\ Information, Shaoyang University, \\ Shaoyang 422004, Hunan, People's \\ Republic of China
}

\begin{abstract}
This paper is concerned with the oscillatory behavior of a certain class of third-order nonlinear variable delay neutral functional dynamic equations,

$$
\left\{r(t) \phi\left(\left[a(t) y^{\Delta}(t)\right]^{\Delta}\right)\right\}^{\Delta}+P(t) F(\phi(x(\delta(t))))=0,
$$

on a time scale $\mathbf{T}$ with sup $\mathbf{T}=+\infty$, where $y(t)=x(t)+B(t) g(x(\tau(t))), \phi(u)=|u|^{\lambda-1} u$, $\lambda \geq 1$. By using the generalized Riccati transformation and a lot of inequality techniques, some new oscillation criteria for the equations are established, results are presented that not only complement and improve those related results in the literature, but also improve some known results for a third-order delay dynamic equation with a neutral term. Further, the main results improve some related results for third-order neutral differential equations. Some examples are given to illustrate the importance of our results.
\end{abstract}

MSC: 34K11;34C10; 39A10

Keywords: oscillation; delay dynamic equations; Riccati transformation; inequality techniques; time scales

\section{Introduction}

Consider a third-order nonlinear variable delay dynamic equation

$$
\left\{r(t) \phi\left(\left[a(t) y^{\Delta}(t)\right]^{\Delta}\right)\right\}^{\Delta}+P(t) F(\phi(x(\delta(t))))=0, \quad t \in \mathbf{T}, t \geq t_{0},
$$

where $y(t)=x(t)+B(t) g(x(\tau(t))), \phi(u)=|u|^{\lambda-1} u, \lambda \geq 1$. Throughout this article, we assume that:

$\left(\mathrm{H}_{1}\right): \mathbf{T}$ is an arbitrary time scale with $\sup \mathbf{T}=+\infty$, and $t_{0} \in \mathbf{T}$ with $t_{0}>0$, we define the time scale interval $\left[t_{0},+\infty\right)_{\mathbf{T}}$ by $\left[t_{0},+\infty\right)_{\mathbf{T}}=\left[t_{0},+\infty\right) \cap \mathbf{T} . r(t), a(t), B(t), P(t) \in$ $C_{\mathrm{rd}}(\mathbf{T}, \mathbf{R})$, i.e., $r(t), a(t), B(t), P(t): \mathbf{T} \rightarrow \mathbf{R}$ are rd-continuous functions. $g(u), F(u)$ : $\mathbf{R} \rightarrow \mathbf{R}$ are continuous functions with $u g(u)>0(u \neq 0)$ and $u F(u)>0(u \neq 0)$.

$\left(\mathrm{H}_{2}\right): \tau(t), \delta(t): \mathbf{T} \rightarrow \mathbf{T}$ are delay functions with $\tau(t) \leq t, \lim _{t \rightarrow+\infty} \tau(t)=+\infty$ and $\delta(t) \leq t$, $\lim _{t \rightarrow+\infty} \delta(t)=+\infty$

$\left(\mathrm{H}_{3}\right): 0 \leq B(t) \leq 1, P(t)>0, r(t)>0$ and $r^{\Delta}(t) \geq 0, a(t)>0$ and $a^{\Delta}(t) \geq 0$.

$\left(\mathrm{H}_{4}\right)$ : There exist constants $0<\beta \leq 1$ and $L>0$, such that $\frac{g(u)}{u} \leq \beta(u \neq 0), \frac{F(u)}{u} \geq L(u \neq 0)$.

$\left(\mathrm{H}_{5}\right): \int_{t_{0}}^{+\infty} \frac{1}{a(s)} \Delta s=+\infty, \int_{t_{0}}^{+\infty} \frac{1}{[r(s)]^{1 / \lambda}} \Delta s=+\infty$. 
We recall that a solution $x(t)$ of equation (1.1) is said to be oscillatory on $\left[t_{0},+\infty\right)_{\mathrm{T}}$ if it is neither eventually positive nor eventually negative; otherwise, the solution is said to be nonoscillatory. Equation (1.1) is said to be oscillatory if all of its solutions are oscillatory. Our attention is restricted to those solutions $x(t)$ of $(1.1)$ where $x(t)$ is not eventually identically zero.

Note that if $\lambda=1, r(t) \equiv 1, a(t) \equiv 1, F(u)=u, B(t) \equiv 0, \delta(t)=t$ in equation (1.1), then (1.1) is simplified to the equation

$$
x^{\Delta \Delta \Delta}(t)+P(t) x(t)=0, \quad t \in \mathbf{T}, t \geq t_{0} .
$$

In equation (1.1), if $r(t) \equiv 1, a(t) \equiv 1, F(u)=u, B(t) \equiv 0$ and $\lambda$ is the ratio of positive odd integers, then (1.1) is simplified to the Emden-Fowler type equation

$$
\left[\left(x^{\Delta \Delta}(t)\right)^{\lambda}\right]^{\Delta}+P(t) x^{\lambda}(\delta(t))=0, \quad t \in \mathbf{T}, t \geq t_{0} .
$$

In equation (1.1), if $\lambda=1, a(t) \equiv 1, F(u)=u^{\gamma}$ (where $\gamma$ is the ratio of positive odd integers), then (1.1) is simplified to the equation

$$
\left[r(t)(x(t)+B(t) x(\tau(t)))^{\Delta \Delta}\right]^{\Delta}+P(t) x^{\gamma}(\delta(t))=0, \quad t \in \mathbf{T}, t \geq t_{0} .
$$

And it is easy to see that (1.1) can be transformed into the third-order nonlinear delay dynamic equations

$$
\left\{r(t)\left[a(t) x^{\Delta}(t)\right]^{\Delta}\right\}^{\Delta}+P(t) f(x(\delta(t)))=0, \quad t \in \mathbf{T}, t \geq t_{0}
$$

and

$$
\left\{r(t)\left[\left(a(t) x^{\Delta}(t)\right)^{\Delta}\right]^{\lambda}\right\}^{\Delta}+P(t) f(x(\delta(t)))=0, \quad t \in \mathbf{T}, t \geq t_{0} .
$$

Equations of this type arise in a number of important applications such as problems in biological population dynamics, in neural network, in quantum theory, in computer science and in control theory. Hence, it is important and useful to study the oscillatory properties of solutions of equation (1.1). Recently, there has been an increasing interest in studying the oscillatory behavior of first and second-order dynamic equations on time scales (see [1-7]). However, there are very few results regarding the oscillation of thirdorder equations. Among these papers dealing with the subject, we refer in particular to [8$17]$, the monographs $[1,2]$ and the references therein. Our concern is especially motivated by several recent papers such as [9-13].

Erbe et al. [9] studied equation (1.2) and they established Hille and Nehari-type oscillation criteria for the equation. After that, in [10] and [11], the author discussed oscillatory criteria of equations (1.3) and (1.4), respectively, under the condition

$$
\int_{t_{0}}^{+\infty} P(u)\left[h_{2}\left(\delta(u), t_{0}\right)\right]^{\lambda} \Delta u=+\infty
$$


and got the result that every solution of equations (1.3) and (1.4) oscillates or converges to zero, where the Taylor monomials $\left\{h_{n}(t, s)\right\}_{n=0}^{+\infty}$ are defined as follows:

$$
h_{0}(t, s)=1, \quad h_{n+1}(t, s)=\int_{s}^{t} h_{n}(\tau, s) \Delta \tau, \quad n \geq 0, t, s \in \mathbf{T} .
$$

In [12], the author discussed oscillatory criteria of equation (1.5) under the conditions

$$
a^{\Delta}(t) \leq 0 \quad \text { and } \quad \int_{t_{0}}^{+\infty} P(u) \delta(u) \Delta u=+\infty
$$

and got the result that every solution of equation (1.5) oscillates or converges to zero. Moreover, in [13], the author considered the oscillation for equation (1.6) under the conditions

$$
\delta \circ \sigma=\sigma \circ \delta, \quad a^{\Delta}(t) \leq 0 \quad \text { and } \quad \int_{t_{0}}^{+\infty} P(u)[\delta(u)]^{\lambda} \Delta u=+\infty
$$

and obtained the result that every solution of equation (1.6) oscillates or converges to zero.

Obviously, the results in [9-13] are inapplicable for the following differential equation

$$
\left\{t^{2}\left[\left(t x^{\prime}(t)\right)^{\prime}\right]^{3}\right\}^{\prime}+t(t-1)^{2}\left(3 t^{2}-8 t+2\right) e^{6 t-18} x^{9}(t-2)=0 .
$$

Therefore, this topic is fairly new for dynamic equations on time scales. The purpose of this article is to obtain new oscillation criteria for the oscillation of (1.1), these criteria can improve the restriction of the conditions for the equation, which promote some existing results. We should note that many of our results of this article are new for the corresponding third-order nonlinear differential and difference equations. In fact, the obtained results extend, unify and correlate many of the existing results in the literature.

\section{Preliminaries}

We shall employ the following lemmas.

Lemma 2.1 [1] Suppose that $x(t)$ is delta-differentiable and eventually positive or eventually negative, then

$$
\left((x(t))^{\lambda}\right)^{\Delta}=\lambda \int_{0}^{1}\left[h x^{\sigma}+(1-h) x\right]^{\lambda-1} x^{\Delta}(t) d h .
$$

Lemma 2.2 [3] Assume that:

(1) $u \in C_{\mathrm{rd}}^{2}(\mathbf{I}, \mathbf{R})$, where $\mathbf{I}=\left[t^{*},+\infty\right), t^{*}>0$.

(2) $u(t)>0, u^{\Delta}(t)>0, u^{\Delta \Delta}(t) \leq 0, t \geq t^{*}$.

Then, for every $k \in(0,1)$, there exists a constant $t_{k} \in \mathbf{T}, t_{k}>t^{*}$, such that $u(\sigma(t)) \leq \frac{\sigma(t) u(\delta(t))}{k \delta(t)}$ $\left(t \geq t_{k}\right)$.

Lemma 2.3 [2] Suppose that $a$ and $b$ are nonnegative real numbers, then $r a b^{r-1}-a^{r} \leq$ $(r-1) b^{r}$ for all $r>1$, where the equality holds if and only if $a=b$. 
Lemma 2.4 [8] Assume that $u(t)>0, u^{\Delta}(t)>0, u^{\Delta \Delta}(t)>0, u^{\Delta \Delta \Delta}(t)<0$, then

$$
\liminf _{t \rightarrow+\infty} \frac{t u(t)}{h_{2}\left(t, t_{0}\right) u^{\Delta}(t)} \geq 1
$$

Lemma 2.5 Let $x(t)$ be an eventually position solution of equation (1.1). Then there exists $t_{1} \in\left[t_{0},+\infty\right)_{\mathrm{T}}$, for all $t \in\left[t_{1},+\infty\right)_{\mathrm{T}}$, such that either

(i) $y(t)>0, y^{\Delta}(t)>0,\left[a(t) y^{\Delta}(t)\right]^{\Delta}>0,\left\{r(t) \phi\left(\left[a(t) y^{\Delta}(t)\right]^{\Delta}\right)\right\}^{\Delta}<0$, or

(ii) $y(t)>0, y^{\Delta}(t)<0,\left[a(t) y^{\Delta}(t)\right]^{\Delta}>0,\left\{r(t) \phi\left(\left[a(t) y^{\Delta}(t)\right]^{\Delta}\right)\right\}^{\Delta}<0$.

Proof Since $x(t)$ is an eventually position solution of (1.1), then there exists $t_{1} \in\left[t_{0},+\infty\right)_{\mathrm{T}}$ such that $x(t)>0, x(\tau(t))>0, x(\delta(t))>0$ for all $t \in\left[t_{1},+\infty\right)_{\mathrm{T}}$, thus, $y(t)>0$. From (1.1), we obtain

$$
\left\{r(t) \phi\left(\left[a(t) y^{\Delta}(t)\right]^{\Delta}\right)\right\}^{\Delta}=-P(t) F(\phi(x(\delta(t)))) \leq-L P(t)(x(\delta(t)))^{\lambda}<0 .
$$

Hence, $r(t) \phi\left(\left[a(t) y^{\Delta}(t)\right]^{\Delta}\right)$ is decreasing and, therefore, eventually of one sign, so $[a(t) \times$ $\left.y^{\Delta}(t)\right]^{\Delta}$ is either eventually positive or eventually negative. We assert that $\left[a(t) y^{\Delta}(t)\right]^{\Delta}>$ 0 for all $t \in\left[t_{1},+\infty\right)_{\mathrm{T}}$. Assume that $\left[a(t) y^{\Delta}(t)\right]^{\Delta}<0$ eventually, then there exists $t_{2} \in$ $\left[t_{1},+\infty\right)_{\mathrm{T}}$, such that $\left[a\left(t_{2}\right) y^{\Delta}\left(t_{2}\right)\right]^{\Delta}<0$. Then for all $t \in\left[t_{2},+\infty\right)_{\mathrm{T}}$, we obtain

$$
r(t) \phi\left(\left[a(t) y^{\Delta}(t)\right]^{\Delta}\right) \leq r\left(t_{2}\right) \phi\left(\left[a\left(t_{2}\right) y^{\Delta}\left(t_{2}\right)\right]^{\Delta}\right)=-M<0,
$$

where $M=-r\left(t_{2}\right) \phi\left(\left[a\left(t_{2}\right) y^{\Delta}\left(t_{2}\right)\right]^{\Delta}\right)=r\left(t_{2}\right)\left|\left[a\left(t_{2}\right) y^{\Delta}\left(t_{2}\right)\right]^{\Delta}\right|^{\lambda-1}\left\{-\left[a\left(t_{2}\right) y^{\Delta}\left(t_{2}\right)\right]^{\Delta}\right\}>0$. By (2.3), we obtain

$$
\left(-\left[a(t) y^{\Delta}(t)\right]^{\Delta}\right)^{\lambda} \geq \frac{M}{r(t)}, \quad \text { namely, } \quad\left[a(t) y^{\Delta}(t)\right]^{\Delta} \leq-M^{1 / \lambda} \frac{1}{[r(t)]^{1 / \lambda}} .
$$

Integrating this inequality from $t_{2}$ to $t\left(t \in\left[t_{2},+\infty\right)_{\mathrm{T}}\right)$ provides

$$
a(t) y^{\Delta}(t) \leq a\left(t_{2}\right) y^{\Delta}\left(t_{2}\right)-M^{1 / \lambda} \int_{t_{2}}^{t} \frac{1}{[r(s)]^{1 / \lambda}} \Delta s \rightarrow-\infty \quad(t \rightarrow+\infty) .
$$

Then there exists $t_{3} \in\left[t_{2},+\infty\right)_{\mathrm{T}}$, such that $a(t) y^{\Delta}(t) \leq a\left(t_{3}\right) y^{\Delta}\left(t_{3}\right)<0$. Similarly, we can get

$$
y(t) \leq y\left(t_{3}\right)+a\left(t_{3}\right) y^{\Delta}\left(t_{3}\right) \int_{t_{3}}^{t} \frac{1}{a(s)} \Delta s \rightarrow-\infty \quad(t \rightarrow+\infty),
$$

which contradicts with $y(t)>0$. So, $\left[a(t) y^{\Delta}(t)\right]^{\Delta}>0$, this implies that $y^{\Delta}(t)>0$ or $y^{\Delta}(t)<0$. This completes the proof.

Lemma 2.6 Assume that $x(t)$ is a solution of equation (1.1), which satisfies the case (ii) in Lemma 2.5, if either

$$
\int_{t_{0}}^{+\infty} P(s) \Delta s=+\infty
$$


or

$$
\begin{aligned}
& \int_{t_{0}}^{+\infty} P(s) \Delta s<+\infty \text { and } \\
& \int_{t_{0}}^{+\infty}\left[\frac{1}{a(v)} \int_{v}^{+\infty}\left(\frac{1}{r(u)} \int_{u}^{+\infty} P(s) \Delta s\right)^{1 / \lambda} \Delta u\right] \Delta v=+\infty
\end{aligned}
$$

holds, then $\lim _{t \rightarrow+\infty} x(t)=0$.

Proof Since $x(t)$ is a solution of equation (1.1), which satisfies the case (ii) in Lemma 2.5, i.e.,

$$
\begin{aligned}
& y(t)>0, \quad y^{\Delta}(t)<0, \quad\left[a(t) y^{\Delta}(t)\right]^{\Delta}>0, \\
& \left\{r(t) \phi\left(\left[a(t) y^{\Delta}(t)\right]^{\Delta}\right)\right\}^{\Delta}<0, \quad t \in\left[t_{1},+\infty\right)_{\mathrm{T}} .
\end{aligned}
$$

Therefore, it follows that $\lim _{t \rightarrow+\infty} y(t)=c \geq 0$. If $c>0$, then in view of $y(t) \leq x(t)+$ $\beta B(t) x(\tau(t))$ and $0 \leq \beta B(t) \leq 1$, it is easy to see that there exists $t_{2} \in\left[t_{1},+\infty\right)_{\mathrm{T}}$ such that $x(\delta(t)) \geq \frac{c}{2}$ for all $t \in\left[t_{2},+\infty\right)_{\mathrm{T}}$. Therefore, from (2.2), we obtain

$$
\left\{r(t) \phi\left(\left[a(t) y^{\Delta}(t)\right]^{\Delta}\right)\right\}^{\Delta} \leq-L P(t)(x(\delta(t)))^{\lambda} \leq-L\left(\frac{c}{2}\right)^{\lambda} P(t) .
$$

If (2.4) holds, then integrating (2.6) from $t_{2}$ to $t\left(t \in\left[t_{2},+\infty\right)_{\mathrm{T}}\right)$ provides

$$
r(t) \phi\left(\left[a(t) y^{\Delta}(t)\right]^{\Delta}\right) \leq r\left(t_{2}\right) \phi\left(\left[a\left(t_{2}\right) y^{\Delta}\left(t_{2}\right)\right]^{\Delta}\right)-L\left(\frac{c}{2}\right)^{\lambda} \int_{t_{2}}^{t} P(s) \Delta s \rightarrow-\infty \quad(t \rightarrow+\infty),
$$

which contradicts with $\left[a(t) y^{\Delta}(t)\right]^{\Delta}>0$. So $\lim _{t \rightarrow+\infty} y(t)=0$, in view of $0<x(t) \leq y(t)$, hence $\lim _{t \rightarrow+\infty} x(t)=0$.

If (2.5) holds, then integrating (2.6) from $t$ to $T\left(T \geq t, T, t \in\left[t_{2},+\infty\right)_{\mathrm{T}}\right)$ provides

$$
r(T) \phi\left(\left[a(T) y^{\Delta}(T)\right]^{\Delta}\right)-r(t) \phi\left(\left[a(t) y^{\Delta}(t)\right]^{\Delta}\right) \leq-L\left(\frac{c}{2}\right)^{\lambda} \int_{t}^{T} P(s) \Delta s
$$

i.e., $r(t) \phi\left(\left[a(t) y^{\Delta}(t)\right]^{\Delta}\right) \geq L\left(\frac{c}{2}\right)^{\lambda} \int_{t}^{T} P(s) \Delta s$, letting $T \rightarrow+\infty$, we have $r(t) \phi\left(\left[a(t) y^{\Delta}(t)\right]^{\Delta}\right) \geq$ $L\left(\frac{c}{2}\right)^{\lambda} \int_{t}^{+\infty} P(s) \Delta s$, this implies that $\left[a(t) y^{\Delta}(t)\right]^{\Delta} \geq L^{1 / \lambda} \frac{c}{2}\left(\frac{1}{r(t)} \int_{t}^{+\infty} P(s) \Delta s\right)^{1 / \lambda}, t \in\left[t_{2},+\infty\right)_{\mathbf{T}}$. In view of $y^{\Delta}(t)<0$, similarly, we can get

$$
y^{\Delta}(t) \leq-L^{1 / \lambda} \frac{c}{2} \frac{1}{a(t)} \int_{t}^{+\infty}\left(\frac{1}{r(u)} \int_{u}^{+\infty} P(s) \Delta s\right)^{1 / \lambda} \Delta u
$$

Integrating the above inequality from $t_{2}$ to $t\left(t \in\left[t_{2},+\infty\right)_{\mathrm{T}}\right)$, we obtain

$$
y(t) \leq y\left(t_{2}\right)-L^{\frac{1}{\lambda}} \frac{c}{2} \int_{t_{2}}^{t}\left[\frac{1}{a(v)} \int_{v}^{+\infty}\left(\frac{1}{r(u)} \int_{u}^{+\infty} P(s) \Delta s\right)^{1 / \lambda} \Delta u\right] \Delta v \rightarrow-\infty \quad(t \rightarrow+\infty),
$$

which contradicts with $y(t)>0$. So, $\lim _{t \rightarrow+\infty} y(t)=0$, further, $\lim _{t \rightarrow+\infty} x(t)=0$. This completes the proof. 
Due to the above reasons, in the next section, we assume that either (2.4) or (2.5) holds.

Lemma 2.7 [17] (Hölder's inequality) Let $a, b \in \mathbf{T}$ and $a<b$. For $r d$-continuous functions $f, g:[a, b] \rightarrow \mathbf{R}$, we have $\int_{a}^{b}|f(u) g(u)| \Delta u \leq\left(\int_{a}^{b}|f(u)|^{p} \Delta u\right)^{\frac{1}{p}}\left(\int_{a}^{b}|g(u)|^{q} \Delta u\right)^{\frac{1}{q}}$, where $p>1$ and $\frac{1}{p}+\frac{1}{q}=1$.

\section{Main results}

In this section, we establish some sufficient conditions which guarantee that every solution $x(t)$ of (1.1) either oscillates on $\left[t_{0},+\infty\right)_{\mathrm{T}}$ or converges as $t \rightarrow+\infty$.

Theorem 3.1 If there exists a function $\varphi(t) \in C_{\mathrm{rd}}^{1}(\mathbf{T},(0,+\infty))$ with $\varphi^{\Delta}(t) \geq 0$, such that

$$
\limsup _{t \rightarrow+\infty} \int_{t_{0}}^{t}\left\{L \varphi(\sigma(s)) P(s) \Psi(s)-\frac{\varphi(s) r(s)}{k^{\lambda^{2}}(\lambda+1)^{\lambda+1}}\left[\frac{\varphi^{\Delta}(s)}{\varphi(s)}\right]^{\lambda+1}\left[\frac{\sigma(s)}{\delta(s)}\right]^{\lambda^{2}}\right\} \Delta s=+\infty
$$

where $\Psi(t)=\frac{k^{\lambda}[1-\beta B(\delta(t))]^{\lambda}\left[h_{2}\left(\delta(t), t_{0}\right)\right]^{\lambda}}{2^{\lambda}[a(\delta(t)) \sigma(t)]^{\lambda}}$, then every solution $x(t)$ of equation $(1.1)$ is either oscillatory or $\lim _{t \rightarrow+\infty} x(t)=0$.

Proof Suppose that equation (1.1) has a nonoscillatory solution $x(t)$ on $\left[t_{0},+\infty\right)_{\mathrm{T}}$. We may assume without loss of generality that $x(t)>0$ and $x(\tau(t))>0, x(\delta(t))>0$ for all $t \in\left[t_{1},+\infty\right)_{\mathrm{T}}, t_{1} \in\left[t_{0},+\infty\right)_{\mathrm{T}}$. Then, by Lemma 2.5 , we see that $x(t)$ satisfies either case (i) or case (ii).

If case (i) in Lemma 2.5 holds, then in view of $x(t) \leq y(t)$, we have

$$
y(t) \leq x(t)+\beta B(t) x(\tau(t)) \leq x(t)+\beta B(t) y(\tau(t)) \leq x(t)+\beta B(t) y(t)
$$

i.e.,

$$
x(t) \geq[1-\beta B(t)] y(t) .
$$

Now define the function $V(t)$ by

$$
V(t)=\varphi(t) \frac{r(t) \phi\left(\left[a(t) y^{\Delta}(t)\right]^{\Delta}\right)}{\phi\left(a(t) y^{\Delta}(t)\right)}=\varphi(t) \frac{r(t)\left[\left(a(t) y^{\Delta}(t)\right)^{\Delta}\right]^{\lambda}}{\left(a(t) y^{\Delta}(t)\right)^{\lambda}}, \quad t \in\left[t_{1},+\infty\right)_{\mathrm{T}} .
$$

Then $V(t)>0\left(t \in\left[t_{1},+\infty\right)_{\mathrm{T}}\right)$. In view of (2.2) and (3.3), we obtain for $t \in\left[t_{1},+\infty\right)_{\mathrm{T}}$,

$$
\begin{aligned}
V^{\Delta}(t)= & \varphi^{\Delta}(t) \frac{r(t) \phi\left(\left[a(t) y^{\Delta}(t)\right]^{\Delta}\right)}{\phi\left(a(t) y^{\Delta}(t)\right)}+\varphi(\sigma(t)) \\
& \times \frac{\left\{r(t)\left[\left(a(t) y^{\Delta}(t)\right)^{\Delta}\right]^{\lambda}\right\}^{\Delta}\left(a(t) y^{\Delta}(t)\right)^{\lambda}-r(t)\left[\left(a(t) y^{\Delta}(t)\right)^{\Delta}\right]^{\lambda}\left[\left(a(t) y^{\Delta}(t)\right)^{\lambda}\right]^{\Delta}}{\left(a(t) y^{\Delta}(t)\right)^{\lambda}\left(a(\sigma(t)) y^{\Delta}(\sigma(t))\right)^{\lambda}} \\
\leq & \frac{\varphi^{\Delta}(t)}{\varphi(t)} V(t)-\varphi(\sigma(t)) \\
& \times\left\{\frac{L P(t)(x(\delta(t)))^{\lambda}}{\left(a(\sigma(t)) y^{\Delta}(\sigma(t))\right)^{\lambda}}+\frac{r(t)\left[\left(a(t) y^{\Delta}(t)\right)^{\Delta}\right]^{\lambda}\left[\left(a(t) y^{\Delta}(t)\right)^{\lambda}\right]^{\Delta}}{\left(a(t) y^{\Delta}(t)\right)^{\lambda}\left(a(\sigma(t)) y^{\Delta}(\sigma(t))\right)^{\lambda}}\right\} .
\end{aligned}
$$


Now, let $u(t)=a(t) y^{\Delta}(t)$, then from (i) in Lemma 2.5, we have $u(t)>0, u^{\Delta}(t)>0$. In view of that

$$
\left\{r(t)\left[a(t) y^{\Delta}(t)\right]^{\Delta}\right\}^{\Delta}=\left\{r(t) u^{\Delta}(t)\right\}^{\Delta}=r^{\Delta}(t) u^{\Delta}(\sigma(t))+r(t) u^{\Delta \Delta}(t)<0
$$

and $r^{\Delta}(t) \geq 0$, it is not difficult to see that $u^{\Delta \Delta}(t)<0$. Therefore, by Lemma 2.2, $\forall k \in$ $(0,1), \exists t_{2} \in\left[t_{1},+\infty\right)_{\mathrm{T}}$ with $t_{2} \geq \max \left\{t_{k}, t_{1}\right\}$, such that $u(\sigma(t)) \leq \frac{\sigma(t) u(\delta(t))}{k \delta(t)} \leq \frac{\sigma(t) u(t)}{k \delta(t)}$ for all $t \in\left[t_{2},+\infty\right)_{\mathrm{T}}$, this implies that

$$
a(\sigma(t)) y^{\Delta}(\sigma(t)) \leq \frac{\sigma(t) a(\delta(t)) y^{\Delta}(\delta(t))}{k \delta(t)} \leq \frac{\sigma(t) a(t) y^{\Delta}(t)}{k \delta(t)} .
$$

From (2.1), we get $\left[(u(t))^{\lambda}\right]^{\Delta} \geq \lambda \int_{0}^{1}[h u+(1-h) u]^{\lambda-1} u^{\Delta}(t) d h=\lambda(u(t))^{\lambda-1} u^{\Delta}(t)$, therefore,

$$
\left[\left(a(t) y^{\Delta}(t)\right)^{\lambda}\right]^{\Delta} \geq \lambda\left(a(t) y^{\Delta}(t)\right)^{\lambda-1}\left(a(t) y^{\Delta}(t)\right)^{\Delta} .
$$

Using (3.2), (3.5), and (3.6) in (3.4), we find

$$
\begin{aligned}
V^{\Delta}(t) \leq & \frac{\varphi^{\Delta}(t)}{\varphi(t)} V(t)-\varphi(\sigma(t)) \\
& \times\left\{\frac{L P(t)[1-\beta B(\delta(t))]^{\lambda}(y(\delta(t)))^{\lambda}}{\left(a(\sigma(t)) y^{\Delta}(\sigma(t))\right)^{\lambda}}+\frac{\lambda(k \delta(t))^{\lambda} r(t)\left[\left(a(t) y^{\Delta}(t)\right)^{\Delta}\right]^{\lambda+1}}{(\sigma(t))^{\lambda}\left[a(t) y^{\Delta}(t)\right]^{\lambda+1}}\right\} \\
= & \frac{\varphi^{\Delta}(t)}{\varphi(t)} V(t)-\varphi(\sigma(t)) \frac{L P(t)[1-\beta B(\delta(t))]^{\lambda}(y(\delta(t)))^{\lambda}}{\left(a(\sigma(t)) y^{\Delta}(\sigma(t))\right)^{\lambda}} \\
& -\frac{\lambda k^{\lambda} \varphi(\sigma(t)) r(t) \delta^{\lambda}(t)[V(t)]^{\frac{\lambda+1}{\lambda}}}{[r(t) \varphi(t)]^{(\lambda+1) / \lambda}(\sigma(t))^{\lambda}} \\
\leq & \frac{\varphi^{\Delta}(t)}{\varphi(t)} V(t)-\varphi(\sigma(t)) \frac{L P(t)[1-\beta B(\delta(t))]^{\lambda}(y(\delta(t)))^{\lambda}}{\left(a(\sigma(t)) y^{\Delta}(\sigma(t))\right)^{\lambda}} \\
& -\frac{\lambda k^{\lambda} \delta^{\lambda}(t)[V(t)]^{\frac{\lambda+1}{\lambda}}}{[r(t) \varphi(t)]^{1 / \lambda} \sigma^{\lambda}(t)} .
\end{aligned}
$$

On the other hand, let $U(t)=\int_{t_{1}}^{t} a(s) y^{\Delta}(s) \Delta s\left(t \in\left(t_{1},+\infty\right)_{\mathrm{T}}\right)$, similarly, it is easy to see that $U(t)>0, U^{\Delta}(t)>0, U^{\Delta \Delta}(t)>0, U^{\Delta \Delta \Delta}(t)<0$. Therefore, by Lemma $2.4, \exists t_{1 / 2} \in\left(t_{1},+\infty\right)_{\mathrm{T}}$, such that $\frac{t U(t)}{h_{2}\left(t, t_{0}\right) U^{\Delta}(t)} \geq \frac{1}{2}$ for all $t \in\left[t_{1 / 2},+\infty\right)_{\mathrm{T}}$. Then we see that

$$
\frac{\int_{t_{1}}^{t} a(s) y^{\Delta}(s) \Delta s}{a(t) y^{\Delta}(t)} \geq \frac{h_{2}\left(t, t_{0}\right)}{2 t}, \quad t \in\left[t_{1 / 2},+\infty\right)_{\mathrm{T}}
$$

From $\int_{t_{1}}^{t} a(s) y^{\Delta}(s) \Delta s=a(t) y(t)-a\left(t_{1}\right) y\left(t_{1}\right)-\int_{t_{1}}^{t} a^{\Delta}(s) y(\sigma(s)) \Delta s$, we get $a(t) y(t) \geq \int_{t_{1}}^{t} a(s) \times$ $y^{\Delta}(s) \Delta s$. In view of (3.8), we then obtain

$$
\frac{y(t)}{y^{\Delta}(t)}=\frac{a(t) y(t)}{a(t) y^{\Delta}(t)} \geq \frac{\int_{t_{1}}^{t} a(s) y^{\Delta}(s) \Delta s}{a(t) y^{\Delta}(t)} \geq \frac{h_{2}\left(t, t_{0}\right)}{2 t}, \quad t \in\left[t_{1 / 2},+\infty\right) \mathrm{T}
$$


Therefore, by (3.5) and (3.9), $\exists T_{0} \in\left[t_{0},+\infty\right)_{\mathrm{T}}$ with $T_{0} \geq \max \left\{t_{2}, t_{1 / 2}\right\}$, such that

$$
\begin{aligned}
\frac{y(\delta(t))}{a(\sigma(t)) y^{\Delta}(\sigma(t))} & =\frac{1}{a(\sigma(t))} \frac{y(\delta(t))}{y^{\Delta}(\delta(t))} \frac{y^{\Delta}(\delta(t))}{y^{\Delta}(\sigma(t))} \\
& \geq \frac{1}{a(\sigma(t))} \frac{h_{2}\left(\delta(t), t_{0}\right)}{2 \delta(t)} \frac{k \delta(t) a(\sigma(t))}{\sigma(t) a(\delta(t))}=\frac{k h_{2}\left(\delta(t), t_{0}\right)}{2 a(\delta(t)) \sigma(t)}
\end{aligned}
$$

for all $t \in\left[T_{0},+\infty\right)_{\mathrm{T}}$. Using the above inequality in (3.7), we obtain for all $t \in\left[T_{0},+\infty\right)_{\mathrm{T}}$,

$$
\begin{aligned}
V^{\Delta}(t) \leq & -\varphi(\sigma(t)) \frac{L P(t)[1-\beta B(\delta(t))]^{\lambda}\left[k h_{2}\left(\delta(t), t_{0}\right)\right]^{\lambda}}{[2 a(\delta(t)) \sigma(t)]^{\lambda}}+\frac{\varphi^{\Delta}(t)}{\varphi(t)} V(t) \\
& -\frac{\lambda k^{\lambda} \delta^{\lambda}(t)}{[r(t) \varphi(t)]^{1 / \lambda} \sigma^{\lambda}(t)}[V(t)]^{\lambda+1}
\end{aligned}
$$

i.e.,

$$
L \varphi(\sigma(t)) P(t) \Psi(t) \leq-V^{\Delta}(t)+\frac{\varphi^{\Delta}(t)}{\varphi(t)} V(t)-\frac{\lambda k^{\lambda} \delta^{\lambda}(t)}{[r(t) \varphi(t)]^{1 / \lambda} \sigma^{\lambda}(t)}[V(t)]^{\frac{\lambda+1}{\lambda}} .
$$

In Lemma 2.3, we let

$$
\begin{aligned}
& r=\frac{\lambda+1}{\lambda}, \quad a=\lambda \frac{\lambda}{\lambda+1}\left[\frac{k \delta(t)}{(r(t) \varphi(t))^{1 / \lambda^{2}} \sigma(t)}\right]^{\frac{\lambda^{2}}{\lambda+1}} V(t), \\
& b=\frac{\lambda \frac{\lambda}{\lambda+1}}{(\lambda+1)^{\lambda}}\left[\frac{\varphi^{\Delta}(t)}{\varphi(t)}\right]^{\lambda}\left[\frac{k \delta(t)}{(r(t) \varphi(t))^{1 / \lambda^{2}} \sigma(t)}\right]^{\frac{-\lambda^{3}}{\lambda+1}} .
\end{aligned}
$$

From Lemma 2.3, we then obtain

$$
\frac{\varphi^{\Delta}(t)}{\varphi(t)} V(t)-\frac{\lambda k^{\lambda} \delta^{\lambda}(t)}{[r(t) \varphi(t)]^{1 / \lambda} \sigma^{\lambda}(t)}[V(t)]^{\frac{\lambda+1}{\lambda}} \leq \frac{\varphi(t) r(t)}{k^{\lambda^{2}}(\lambda+1)^{\lambda+1}}\left[\frac{\varphi^{\Delta}(t)}{\varphi(t)}\right]^{\lambda+1}\left[\frac{\sigma(t)}{\delta(t)}\right]^{\lambda^{2}} .
$$

Using the above inequality in (3.10), we find

$$
L \varphi(\sigma(t)) P(t) \Psi(t) \leq-V^{\Delta}(t)+\frac{\varphi(t) r(t)}{k^{\lambda^{2}}(\lambda+1)^{\lambda+1}}\left[\frac{\varphi^{\Delta}(t)}{\varphi(t)}\right]^{\lambda+1}\left[\frac{\sigma(t)}{\delta(t)}\right]^{\lambda^{2}}
$$

Integrating inequality (3.11) from $T_{0}$ to $t \in\left[T_{0},+\infty\right)_{\mathrm{T}}$ provides

$$
\int_{T_{0}}^{t} L \varphi(\sigma(s)) P(s) \Psi(s) \Delta s \leq-V(t)+V\left(T_{0}\right)+\int_{T_{0}}^{t} \frac{\varphi(s) r(s)}{k^{\lambda^{2}}(\lambda+1)^{\lambda+1}}\left[\frac{\varphi^{\Delta}(s)}{\varphi(s)}\right]^{\lambda+1}\left[\frac{\sigma(s)}{\delta(s)}\right]^{\lambda^{2}} \Delta s .
$$

Consequently,

$$
\begin{aligned}
& \int_{T_{0}}^{t}\left\{L \varphi(\sigma(s)) P(s) \Psi(s)-\frac{\varphi(s) r(s)}{k^{\lambda^{2}}(\lambda+1)^{\lambda+1}}\left[\frac{\varphi^{\Delta}(s)}{\varphi(s)}\right]^{\lambda+1}\left[\frac{\sigma(s)}{\delta(s)}\right]^{\lambda^{2}}\right\} \Delta s \\
& \leq-V(t)+V\left(T_{0}\right) \leq V\left(T_{0}\right) .
\end{aligned}
$$

Taking limsup on both sides of the above inequality as $t \rightarrow+\infty$, we obtain a contradiction to condition (3.1). 
If case (ii) in Lemma 2.5 holds, then by Lemma 2.6, we have $\lim _{t \rightarrow+\infty} x(t)=0$. This completes the proof.

Remark 3.1 From Theorem 3.1, we can obtain different conditions for oscillation of all solutions of (1.1) with different choices of $\varphi(t)$. For example, $\varphi(t)=M$ (where $M$ is a constant) or $\varphi(t)=t(k=1 / 2)$. Then we have the following results respectively.

Corollary 3.2 If lim $\sup _{t \rightarrow+\infty} \int_{t_{0}}^{t} \frac{[1-\beta B(\delta(s))]^{\lambda}\left[h_{2}\left(\delta(s), t_{0}\right)\right]^{\lambda}}{[a(\delta(s)) \sigma(s)]^{\lambda}} P(s) \Delta s=+\infty$, then every solution $x(t)$ of equation (1.1) is either oscillatory or $\lim _{t \rightarrow+\infty} x(t)=0$.

Corollary 3.3 If $\lim \sup _{t \rightarrow+\infty} \int_{t_{0}}^{t}\left\{\frac{L[1-\beta B(\delta(s))]^{\lambda}\left[h_{2}\left(\delta(s), t_{0}\right)\right]^{\lambda}}{[4 \sigma(s) a(\delta(s))]^{\lambda}} \sigma(s) P(s)-\frac{r(s)\left[\frac{2 \sigma(s)}{\delta(s)}\right]^{\lambda^{2}}}{(\lambda+1)^{\lambda+1} s^{\lambda}}\right\} \Delta s=+\infty$, then every solution $x(t)$ of equation (1.1) is either oscillatory or $\lim _{t \rightarrow+\infty} x(t)=0$.

Theorem 3.4 If there exists a function $\varphi(t) \in C_{\mathrm{rd}}^{1}(\mathbf{T},(0,+\infty))$ and constant $m \geq 1$, such that

$$
\begin{aligned}
& \limsup _{t \rightarrow+\infty} \frac{1}{t^{m}} \int_{T_{0}}^{t}(t-s)^{m}\{L \varphi(\sigma(s)) P(s) \Psi(s) \\
& \left.-\frac{\varphi(s) r(s)}{k^{\lambda^{2}}(\lambda+1)^{\lambda+1}}\left[\frac{\varphi^{\Delta}(s)}{\varphi(s)}\right]^{\lambda+1}\left[\frac{\sigma(s)}{\delta(s)}\right]^{\lambda^{2}}\right\} \Delta s=+\infty
\end{aligned}
$$

for some constant $T_{0} \geq t_{0}$, where the function $\Psi(s)$ is defined as in Theorem 3.1, then every solution $x(t)$ of equation (1.1) is either oscillatory or $\lim _{t \rightarrow+\infty} x(t)=0$.

Proof Suppose that equation (1.1) has a nonoscillatory solution $x(t)$ on $\left[t_{0},+\infty\right)_{\mathrm{T}}$. We may assume without loss of generality that $x(t)>0$ and $x(\tau(t))>0, x(\delta(t))>0$ for all $t \in\left[t_{1},+\infty\right)_{\mathrm{T}}, t_{1} \in\left[t_{0},+\infty\right)_{\mathrm{T}}$. By Lemma 2.5 there are two possible cases. If case (ii) in Lemma 2.5 holds, then clearly $\lim _{t \rightarrow+\infty} x(t)=0$. If case (i) in Lemma 2.5 holds, we proceed as in the proof of Theorem 3.1 to obtain (3.10). Then from (3.10), we have

$$
L \varphi(\sigma(s)) P(s) \Psi(s) \leq-V^{\Delta}(s)+\frac{\varphi^{\Delta}(s)}{\varphi(s)} V(s)-\frac{\lambda k^{\lambda} \delta^{\lambda}(s)}{[r(s) \varphi(s)]^{1 / \lambda} \sigma^{\lambda}(s)}[V(s)]^{\frac{\lambda+1}{\lambda}}
$$

for all $s \in\left[T_{0},+\infty\right)_{\mathrm{T}}$. Multiplying both sides of the above inequality by $(t-s)^{m}$, and integrating with respect to $s$ from $T_{0}$ to $t\left(t \geq T_{0}\right)$, we can obtain

$$
\begin{aligned}
\int_{T_{0}}^{t} L(t-s)^{m} \varphi(\sigma(s)) P(s) \Psi(s) \Delta s \\
\leq-\int_{T_{0}}^{t}(t-s)^{m} V^{\Delta}(s) \Delta s \\
\quad+\int_{T_{0}}^{t}(t-s)^{m} \frac{\varphi^{\Delta}(s)}{\varphi(s)} V(s) \Delta s-\int_{T_{0}}^{t} \frac{\lambda k^{\lambda}(t-s)^{m} \delta^{\lambda}(s)}{[r(s) \varphi(s)]^{1 / \lambda} \sigma^{\lambda}(s)}[V(s)]^{\frac{\lambda+1}{\lambda}} \Delta s \\
=-\left.\left[(t-s)^{m} V(s)\right]\right|_{T_{0}} ^{t}+\int_{T_{0}}^{t}\left[(t-s)^{m}\right]_{s}^{\Delta} V(\sigma(s)) \Delta s \\
\quad+\int_{T_{0}}^{t}(t-s)^{m} \frac{\varphi^{\Delta}(s)}{\varphi(s)} V(s) \Delta s-\int_{T_{0}}^{t} \frac{\lambda k^{\lambda}(t-s)^{m} \delta^{\lambda}(s)}{[r(s) \varphi(s)]^{1 / \lambda} \sigma^{\lambda}(s)}[V(s)]^{\frac{\lambda+1}{\lambda}} \Delta s
\end{aligned}
$$




$$
\begin{aligned}
\leq & \left(t-T_{0}\right)^{m} V\left(T_{0}\right)+\int_{T_{0}}^{t}(t-s)^{m} \frac{\varphi^{\Delta}(s)}{\varphi(s)} V(s) \Delta s \\
& -\int_{T_{0}}^{t} \frac{\lambda k^{\lambda}(t-s)^{m} \delta^{\lambda}(s)}{[r(s) \varphi(s)]^{1 / \lambda} \sigma^{\lambda}(s)}[V(s)]^{\frac{\lambda+1}{\lambda}} \Delta s .
\end{aligned}
$$

Now, in Lemma 2.3, we let

$$
\begin{aligned}
& r=\frac{\lambda+1}{\lambda}, \quad a=\lambda^{\frac{\lambda}{\lambda+1}}\left\{\frac{k \delta(s)}{[r(s) \varphi(t)]^{1 / \lambda^{2}} \sigma(s)}\right\}^{\frac{\lambda^{2}}{\lambda+1}} V(s) \quad \text { and } \\
& b=\frac{\lambda \frac{\lambda}{\lambda+1}}{(\lambda+1)^{\lambda}}\left[\frac{\varphi^{\Delta}(s)}{\varphi(s)}\right]^{\lambda}\left\{\frac{k \delta(s)}{[r(s) \varphi(s)]^{1 / \lambda^{2}} \sigma(s)}\right\}^{\frac{-\lambda^{3}}{\lambda+1}} .
\end{aligned}
$$

From Lemma 2.3, we then obtain

$$
\frac{\varphi^{\Delta}(s)}{\varphi(s)} V(s)-\frac{\lambda k^{\lambda} \delta^{\lambda}(s)}{[r(s) \varphi(s)]^{1 / \lambda} \sigma^{\lambda}(s)}[V(s)]^{\frac{\lambda+1}{\lambda}} \leq \frac{\varphi(s) r(s)}{k^{\lambda^{2}}(\lambda+1)^{\lambda+1}}\left[\frac{\varphi^{\Delta}(s)}{\varphi(s)}\right]^{\lambda+1}\left[\frac{\sigma(s)}{\delta(s)}\right]^{\lambda^{2}} .
$$

Hence, (3.13) implies

$$
\begin{aligned}
& \int_{T_{0}}^{t} L(t-s)^{m} \varphi(\sigma(s)) P(s) \Psi(s) \Delta s \\
& \quad \leq\left(t-T_{0}\right)^{m} V\left(T_{0}\right)+\int_{T_{0}}^{t} \frac{(t-s)^{m} \varphi(s) r(s)}{k^{\lambda^{2}}(\lambda+1)^{\lambda+1}}\left[\frac{\varphi^{\Delta}(s)}{\varphi(s)}\right]^{\lambda+1}\left[\frac{\sigma(s)}{\delta(s)}\right]^{\lambda^{2}} \Delta s
\end{aligned}
$$

and, therefore,

$$
\begin{aligned}
& \frac{1}{t^{m}} \int_{T_{0}}^{t}(t-s)^{m}\left\{L \varphi(\sigma(s)) P(s) \Psi(s)-\frac{\varphi(s) r(s)}{k^{\lambda^{2}}(\lambda+1)^{\lambda+1}}\left[\frac{\varphi^{\Delta}(s)}{\varphi(s)}\right]^{\lambda+1}\left[\frac{\sigma(s)}{\delta(s)}\right]^{\lambda^{2}}\right\} \Delta s \\
& \quad \leq\left(1-\frac{T_{0}}{t}\right)^{m} V\left(T_{0}\right) .
\end{aligned}
$$

Taking the limit superior as $t \rightarrow+\infty$ in the above inequality, we find

$$
\begin{aligned}
& \limsup _{t \rightarrow+\infty} \frac{1}{t^{m}} \int_{T_{0}}^{t}(t-s)^{m}\left\{L \varphi(\sigma(s)) P(s) \Psi(s)-\frac{\varphi(s) r(s)}{k^{\lambda^{2}}(\lambda+1)^{\lambda+1}}\left[\frac{\varphi^{\Delta}(s)}{\varphi(s)}\right]^{\lambda+1}\left[\frac{\sigma(s)}{\delta(s)}\right]^{\lambda^{2}}\right\} \Delta s \\
& \quad<+\infty
\end{aligned}
$$

contradicting (3.12). This completes the proof.

Remark 3.2 From Theorem 3.4, we can obtain different conditions for oscillation of all solutions of (1.1) with different choices of $\varphi(t)$. For example, $\varphi(t)=M$ (where $M$ is a constant) or $\varphi(t)=t(k=1 / 2)$, then we have the following results, respectively.

Corollary 3.5 If there exists a constant $m \geq 1$, such that

$$
\limsup _{t \rightarrow+\infty} \frac{1}{t^{m}} \int_{T_{0}}^{t}(t-s)^{m} \frac{P(s)[1-\beta B(\delta(s))]^{\lambda}\left[h_{2}\left(\delta(s), t_{0}\right)\right]^{\lambda}}{[\sigma(s) a(\delta(s))]^{\lambda}} \Delta s<+\infty
$$


for some constant $T_{0} \geq t_{0}$, then every solution $x(t)$ of equation (1.1) is either oscillatory or $\lim _{t \rightarrow+\infty} x(t)=0$.

Corollary 3.6 If there exists a constant $m \geq 1$, such that

$$
\begin{aligned}
& \limsup _{t \rightarrow+\infty} \frac{1}{t^{m}} \int_{t_{0}}^{t}(t-s)^{m}\left\{\frac{L[1-\beta B(\delta(s))]^{\lambda}\left[h_{2}\left(\delta(s), t_{0}\right)\right]^{\lambda}}{[4 \sigma(s) a(\delta(s))]^{\lambda}} \sigma(s) P(s)-\frac{r(s)\left[\frac{2 \sigma(s)}{\delta(s)}\right]^{\lambda^{2}}}{(\lambda+1)^{\lambda+1} s^{\lambda}}\right\} \Delta s \\
& \quad=+\infty
\end{aligned}
$$

for some constant $T_{0} \geq t_{0}$, then every solution $x(t)$ of equation (1.1) is either oscillatory or $\lim _{t \rightarrow+\infty} x(t)=0$.

Remark 3.3 Clearly, Kamenev-type oscillation criteria for second-order linear differential equation was extended to third-order nonlinear variable delay dynamic equations on time scales. One can easily see that the recent results cannot be applied in equation (1.1), so our results are new ones.

If (3.12) does not hold, then we have the following result.

Theorem 3.7 If there exist functions $\varphi(t) \in C_{\mathrm{rd}}^{1}(\mathbf{T},(0,+\infty)), \xi(t) \in C_{\mathrm{rd}}(\mathbf{T}, \mathbf{R})$ and a constant $m \geq 1$, such that

$$
\begin{aligned}
& \limsup _{t \rightarrow+\infty} \frac{1}{t^{m}} \int_{T_{0}}^{t}(t-s)^{m} \varphi(s) r(s)\left[\frac{\varphi^{\Delta}(s)}{\varphi(s)}\right]^{\lambda+1}\left[\frac{\sigma(s)}{\delta(s)}\right]^{\lambda^{2}} \Delta s<+\infty \\
& \limsup _{t \rightarrow+\infty} \frac{1}{t^{m}} \int_{u}^{t}(t-s)^{m}\left\{L \varphi(\sigma(s)) P(s) \Psi(s)-\frac{\varphi(s) r(s)}{k^{\lambda^{2}}(\lambda+1)^{\lambda+1}}\left[\frac{\varphi^{\Delta}(s)}{\varphi(s)}\right]^{\lambda+1}\left[\frac{\sigma(s)}{\delta(s)}\right]^{\lambda^{2}}\right\} \Delta s \\
& \quad \geq \xi(u), \quad u \geq T_{0}, \\
& \int_{T_{0}}^{+\infty} \frac{\delta^{\lambda}(s)}{[r(s) \varphi(s)]^{1 / \lambda} \sigma^{\lambda}(s)}[\xi(s)]^{\frac{\lambda+1}{\lambda}} \Delta s=+\infty
\end{aligned}
$$

for some constant $T_{0} \geq t_{0}$, where $\xi_{+}(t)=\max \{\xi(t), 0\}, \Psi(s)$ is defined as in Theorem 3.1, then every solution $x(t)$ of equation (1.1) is either oscillatory or $\lim _{t \rightarrow+\infty} x(t)=0$.

Proof Suppose that equation (1.1) has a nonoscillatory solution $x(t)$ on $\left[t_{0},+\infty\right)_{\mathrm{T}}$. We may assume without loss of generality that $x(t)>0$ and $x(\tau(t))>0, x(\delta(t))>0$ for all $t \in\left[t_{1},+\infty\right)_{\mathrm{T}}, t_{1} \in\left[t_{0},+\infty\right)_{\mathrm{T}}$. By Lemma 2.5 , there are two possible cases. If case (ii) in Lemma 2.5 holds, then clearly $\lim _{t \rightarrow+\infty} x(t)=0$. If case (i) in Lemma 2.5 holds, we proceed as in the proof of Theorem 3.4 to obtain (3.13) and (3.14). Then from (3.14), for $t \geq u \geq T_{0}$, $t, u \in\left[T_{0},+\infty\right)_{\mathrm{T}}$, we have

$$
\begin{aligned}
& \limsup _{t \rightarrow+\infty} \frac{1}{t^{m}} \int_{u}^{t}(t-s)^{m}\left\{L \varphi(\sigma(s)) P(s) \Psi(s)-\frac{\varphi(s) r(s)}{k^{\lambda^{2}}(\lambda+1)^{\lambda+1}}\left[\frac{\varphi^{\Delta}(s)}{\varphi(s)}\right]^{\lambda+1}\left[\frac{\sigma(s)}{\delta(s)}\right]^{\lambda^{2}}\right\} \Delta s \\
& \quad \leq V(u) .
\end{aligned}
$$


In view of (3.16), we can get

$$
\begin{aligned}
& \xi(u) \leq V(u), \quad u \geq T_{0} \\
& \limsup _{t \rightarrow+\infty} \frac{1}{t^{m}} \int_{T_{0}}^{t} L(t-s)^{m} \varphi(\sigma(s)) P(s) \Psi(s) \Delta s \geq \xi\left(T_{0}\right) .
\end{aligned}
$$

From (3.13), we obtain for all $t \in\left[T_{0},+\infty\right)_{\mathrm{T}}$,

$$
\begin{aligned}
\frac{1}{t^{m}} & \int_{T_{0}}^{t} L(t-s)^{m} \varphi(\sigma(s)) P(s) \Psi(s) \Delta s \\
& \leq\left(1-\frac{T_{0}}{t}\right)^{m} V\left(T_{0}\right)+\frac{1}{t^{m}} \int_{T_{0}}^{t}(t-s)^{m} \frac{\varphi^{\Delta}(s)}{\varphi(s)} V(s) \Delta s \\
& -\frac{1}{t^{m}} \int_{T_{0}}^{t} \frac{\lambda k^{\lambda}(t-s)^{m} \delta^{\lambda}(s)}{[r(s) \varphi(s)]^{1 / \lambda} \sigma^{\lambda}(s)}[V(s)]^{\frac{\lambda+1}{\lambda}} \Delta s .
\end{aligned}
$$

Next, in the above inequality, we set

$$
\begin{aligned}
& \varphi_{1}(t)=\frac{1}{t^{m}} \int_{T_{0}}^{t}(t-s)^{m} \frac{\varphi^{\Delta}(s)}{\varphi(s)} V(s) \Delta s \text { and } \\
& \varphi_{2}(t)=\frac{1}{t^{m}} \int_{T_{0}}^{t} \frac{\lambda k^{\lambda}(t-s)^{m} \delta^{\lambda}(s)}{[r(s) \varphi(s)]^{1 / \lambda} \sigma^{\lambda}(s)}[V(s)]^{\frac{\lambda+1}{\lambda}} \Delta s,
\end{aligned}
$$

in view of the second inequality in (3.18), then, it is not difficult to see that

$$
\begin{aligned}
& \liminf _{t \rightarrow+\infty}\left[\varphi_{2}(t)-\varphi_{1}(t)\right] \\
& \quad \leq V\left(T_{0}\right)-\limsup _{t \rightarrow+\infty} \frac{1}{t^{m}} \int_{T_{0}}^{t} L(t-s)^{m} \varphi(\sigma(s)) P(s) \Psi(s) \Delta s \\
& \quad \leq V\left(T_{0}\right)-\xi\left(T_{0}\right)<+\infty
\end{aligned}
$$

Now we claim that

$$
\int_{T_{0}}^{+\infty} \frac{\delta^{\lambda}(s)}{[r(s) \varphi(s)]^{1 / \lambda} \sigma^{\lambda}(s)}[V(s)]^{\frac{\lambda+1}{\lambda}} \Delta s<+\infty
$$

Suppose to the contrary that

$$
\int_{T_{0}}^{+\infty} \frac{\delta^{\lambda}(s)}{[r(s) \varphi(s)]^{1 / \lambda} \sigma^{\lambda}(s)}[V(s)]^{\frac{\lambda+1}{\lambda}} \Delta s=+\infty
$$

Let $M>0$ be arbitrary. Then it follows from the above formula that there exists $t_{2} \in$ $\left[T_{0},+\infty\right)_{\mathrm{T}}$ such that

$$
\int_{T_{0}}^{t} \frac{\delta^{\lambda}(s)}{[r(s) \varphi(s)]^{1 / \lambda} \sigma^{\lambda}(s)}[V(s)]^{\frac{\lambda+1}{\lambda}} \Delta s>\frac{M 2^{m}}{\lambda k^{\lambda}} \quad \text { for } t \in\left[t_{2},+\infty\right)_{\mathbf{T}} .
$$


Therefore (use the integration by parts formula $\int_{a}^{b} f(s) g^{\Delta}(s) \Delta s=[f(s) g(s)]_{a}^{b}-\int_{a}^{b} f^{\Delta}(s) \times$ $g(\sigma(s)) \Delta s)$

$$
\begin{aligned}
\varphi_{2}(t) & =\frac{1}{t^{m}} \int_{T_{0}}^{t} \frac{\lambda k^{\lambda}(t-s)^{m} \delta^{\lambda}(s)}{[r(s) \varphi(s)]^{1 / \lambda} \sigma^{\lambda}(s)}[V(s)]^{\frac{\lambda+1}{\lambda}} \Delta s \\
& =\frac{1}{t^{m}} \int_{T_{0}}^{t}\left\{\lambda k^{\lambda}(t-s)^{m}\left(\int_{T_{0}}^{s} \frac{\delta^{\lambda}(u)}{[r(u) \varphi(u)]^{1 / \lambda} \sigma^{\lambda}(u)}[V(u)]^{\frac{\lambda+1}{\lambda}} \Delta u\right)_{s}^{\Delta}\right\} \Delta s \\
& =\frac{1}{t^{m}} \int_{T_{0}}^{t}\left\{\lambda k^{\lambda}\left[-(t-s)^{m}\right]_{s}^{\Delta} \int_{T_{0}}^{\sigma(s)} \frac{\delta^{\lambda}(u)}{[r(u) \varphi(u)]^{1 / \lambda} \sigma^{\lambda}(u)}[V(u)]^{\frac{\lambda+1}{\lambda}} \Delta u\right\} \Delta s \\
& \geq \frac{1}{t^{m}} \int_{t_{2}}^{t}\left\{\lambda k^{\lambda}\left[-(t-s)^{m}\right]_{s}^{\Delta} \int_{T_{0}}^{s} \frac{\delta^{\lambda}(u)}{[r(u) \varphi(u)]^{1 / \lambda} \sigma^{\lambda}(u)}[V(u)]^{\frac{\lambda+1}{\lambda}} \Delta u\right\} \Delta s \\
& >\frac{1}{t^{m}} \int_{t_{2}}^{t} \lambda k^{\lambda}\left[-(t-s)^{m}\right]_{s}^{\Delta} \frac{M 2^{m}}{\lambda k^{\lambda}} \Delta s=\frac{M 2^{m}}{t^{m}} \int_{t_{2}}^{t}\left[-(t-s)^{m}\right]_{s}^{\Delta} \Delta s=M 2^{m}\left(1-\frac{t_{2}}{t}\right)^{m} .
\end{aligned}
$$

Now, taking $t_{3}=2 t_{2}$, then for $t \in\left[t_{3},+\infty\right)_{\mathrm{T}}, \varphi_{2}(t)>M 2^{m}\left(1-\frac{t_{2}}{t}\right)^{m} \geq M 2^{m}\left(1-\frac{t_{2}}{2 t_{2}}\right)^{m}=M$. Since $M$ is arbitrary,

$$
\lim _{t \rightarrow+\infty} \varphi_{2}(t)=+\infty
$$

Next, we consider a sequence $\left\{T_{n}\right\}_{n=1}^{+\infty}: T_{n} \in\left[T_{0},+\infty\right)_{\mathrm{T}}$ with $\lim _{n \rightarrow+\infty} T_{n}=+\infty$ satisfying

$$
\lim _{n \rightarrow+\infty}\left[\varphi_{2}\left(T_{n}\right)-\varphi_{1}\left(T_{n}\right)\right]=\liminf _{t \rightarrow+\infty}\left[\varphi_{2}(t)-\varphi_{1}(t)\right]<+\infty .
$$

Then there exists a constant $M_{0}>0$ such that

$$
\varphi_{2}\left(T_{n}\right)-\varphi_{1}\left(T_{n}\right) \leq M_{0}
$$

for all sufficiently large positive integer $n$. Since (3.21) ensures that

$$
\lim _{n \rightarrow+\infty} \varphi_{2}\left(T_{n}\right)=+\infty
$$

(3.22) implies that

$$
\lim _{n \rightarrow+\infty} \varphi_{1}\left(T_{n}\right)=+\infty
$$

Furthermore, (3.22) and (3.23) lead to the inequality

$$
\frac{\varphi_{1}\left(T_{n}\right)}{\varphi_{2}\left(T_{n}\right)}-1 \geq-\frac{M_{0}}{\varphi_{2}\left(T_{n}\right)}>-\frac{M_{0}}{2 M_{0}}=-\frac{1}{2}, \quad \text { i.e. } \frac{\varphi_{1}\left(T_{n}\right)}{\varphi_{2}\left(T_{n}\right)}>\frac{1}{2}
$$

for large enough positive integer $n$, which together with (3.24) implies

$$
\lim _{n \rightarrow+\infty} \frac{\left[\varphi_{1}\left(T_{n}\right)\right]^{\lambda+1}}{\left[\varphi_{2}\left(T_{n}\right)\right]^{\lambda}}=\lim _{n \rightarrow+\infty}\left[\frac{\varphi_{1}\left(T_{n}\right)}{\varphi_{2}\left(T_{n}\right)}\right]^{\lambda} \varphi_{1}\left(T_{n}\right)=+\infty .
$$


On the other hand, by Lemma 2.7 , we find that

$$
\begin{aligned}
\varphi_{1}\left(T_{n}\right)= & \frac{1}{T_{n}^{m}} \int_{T_{0}}^{T_{n}}\left(T_{n}-s\right)^{m} \frac{\varphi^{\Delta}(s)}{\varphi(s)} V(s) \Delta s \\
= & \int_{T_{0}}^{T_{n}}\left(\left[\frac{\left(T_{n}-s\right)^{m}}{T_{n}^{m}} \frac{\lambda k^{\lambda} \delta^{\lambda}(s)}{[r(s) \varphi(s)]^{1 / \lambda} \sigma^{\lambda}(s)}\right]^{\frac{\lambda}{\lambda+1}} V(s)\right) \\
& \times\left(\frac{\left(T_{n}-s\right)^{m}}{T_{n}^{m}} \frac{\varphi^{\Delta}(s)}{\varphi(s)}\left[\frac{\left(T_{n}-s\right)^{m}}{T_{n}^{m}} \frac{\lambda k^{\lambda} \delta^{\lambda}(s)}{[r(s) \varphi(s)]^{1 / \lambda} \sigma^{\lambda}(s)}\right]^{\frac{-\lambda}{\lambda+1}}\right) \Delta s \\
\leq & \left\{\int_{T_{0}}^{T_{n}} \frac{\left(T_{n}-s\right)^{m}}{T_{n}^{m}} \frac{\lambda k^{\lambda} \delta^{\lambda}(s)}{[r(s) \varphi(s)]^{1 / \lambda} \sigma^{\lambda}(s)}[V(s)]^{\frac{\lambda+1}{\lambda}} \Delta s\right\}^{\frac{\lambda}{\lambda+1}} \\
& \times\left\{\int_{T_{0}}^{T_{n}}\left[\frac{\left(T_{n}-s\right)^{m}}{T_{n}^{m}} \frac{\varphi^{\Delta}(s)}{\varphi(s)}\right]^{\lambda+1}\left[\frac{\left(T_{n}-s\right)^{m}}{T_{n}^{m}} \frac{\lambda k^{\lambda} \delta^{\lambda}(s)}{[r(s) \varphi(s)]^{1 / \lambda} \sigma^{\lambda}(s)}\right]^{-\lambda} \Delta s\right\}^{\frac{1}{\lambda+1}} \\
= & {\left[\varphi_{2}\left(T_{n}\right)\right]^{\frac{\lambda}{\lambda+1}}\left\{\frac{\left(\lambda k^{\lambda}\right)^{-\lambda}}{T_{n}^{m}} \int_{T_{0}}^{T_{n}}\left(T_{n}-s\right)^{m} r(s) \varphi(s)\left[\frac{\varphi^{\Delta}(s)}{\varphi(s)}\right]^{\lambda+1}\left[\frac{\sigma(s)}{\delta(s)}\right]^{\lambda^{2}} \Delta s\right\}^{\frac{1}{\lambda+1}} }
\end{aligned}
$$

and accordingly,

$$
\frac{\left[\varphi_{1}\left(T_{n}\right)\right]^{\lambda+1}}{\left[\varphi_{2}\left(T_{n}\right)\right]^{\lambda}} \leq \frac{\left(\lambda k^{\lambda}\right)^{-\lambda}}{T_{n}^{m}} \int_{T_{0}}^{T_{n}}\left(T_{n}-s\right)^{m} r(s) \varphi(s)\left[\frac{\varphi^{\Delta}(s)}{\varphi(s)}\right]^{\lambda+1}\left[\frac{\sigma(s)}{\delta(s)}\right]^{\lambda^{2}} \Delta s .
$$

So, because of (3.25), we can obtain

$$
\lim _{n \rightarrow+\infty} \frac{1}{T_{n}^{m}} \int_{T_{0}}^{T_{n}}\left(T_{n}-s\right)^{m} r(s) \varphi(s)\left[\frac{\varphi^{\Delta}(s)}{\varphi(s)}\right]^{\lambda+1}\left[\frac{\sigma(s)}{\delta(s)}\right]^{\lambda^{2}} \Delta s=+\infty,
$$

contradicting (3.15). Therefore, (3.19) holds. Now, from the first formula of (3.18) and (3.19), we get

$$
\int_{T_{0}}^{+\infty} \frac{\delta^{\lambda}(s)}{[r(s) \varphi(s)]^{1 / \lambda} \sigma^{\lambda}(s)}[\xi(s)]^{\frac{\lambda+1}{\lambda}} \Delta s \leq \int_{T_{0}}^{+\infty} \frac{\delta^{\lambda}(s)}{[r(s) \varphi(s)]^{1 / \lambda} \sigma^{\lambda}(s)}[V(s)]^{\frac{\lambda+1}{\lambda}} \Delta s<+\infty,
$$

which contradicts (3.17). This completes the proof.

Obviously, our results in this paper not only extend and improve some known results, and show some results of $[3-8,10,14,15]$ to be special examples of our results, but also unify the oscillation of the third-order nonlinear variable delay differential equations and the third-order nonlinear variable delay difference equations with a nonlinear neutral term. The theorems in this paper are new even for the cases $\mathbf{T}=\mathbf{R}$ and $\mathbf{T}=\mathbf{Z}$.

\section{Applications and examples}

In this section, we give some examples to illustrate our main results.

Example 4.1 Consider third-order delay differential equation

$$
\left\{t^{2}\left[\left(t x^{\prime}(t)\right)^{\prime}\right]^{3}\right\}^{\prime}+t(t-1)^{2}\left(3 t^{2}-8 t+2\right) e^{6 t-18} x^{9}(t-2)=0, \quad t \geq 2 .
$$


It is easy to verify that all conditions of Corollary 3.3 are satisfied. Hence, every solution of equation (4.1) is oscillatory or tends to zero as $t \rightarrow+\infty$. For example, it is not difficult to verify that $x(t)=e^{-t}$ is a solution of equation (4.1). The important point to note here is that the recent results due to $[9-13,15]$ do not apply to equation (4.1) for the condition (1.7) or (1.8) can be a restrictive condition.

Example 4.2 Consider third-order variable delay dynamic equations on time scales

$$
\left\{t^{\frac{2}{5}} \phi\left(\left[t(x(t)+B(t) g(x(\tau(t))))^{\Delta}\right]^{\Delta}\right)\right\}^{\Delta}+P(t) F(\phi(x(\delta(t))))=0, \quad t \in 2^{\mathbf{Z}}, t \geq 2 .
$$

This is a third-order 2-difference equation, here $t_{0}=2, r(t)=t^{\frac{2}{5}}, a(t)=t$. Now, pick $\lambda=\frac{3}{7}$, $B(t)=\frac{1}{\sqrt{2}}-\frac{1}{t}, \tau(t)=\delta(t)=\frac{t}{2}, P(t)=\frac{2^{\frac{3}{4}}}{t^{4 / 7}(t+\sqrt{2})^{3 / 7}}\left[\frac{\sigma(t)}{h_{2}\left(\delta(t), t_{0}\right)}\right]^{3 / 7}, g(u)=\frac{u}{\sqrt{2+\sin ^{4} u}}, f(u)=u[6+$ $\left.\ln \left(1+u^{2}\right)\right]$, then

$$
\begin{aligned}
1-\mu(t) \frac{b(t)}{r(t)} & =1-t \cdot \frac{t^{-2}}{t^{2 / 5}}=1-\frac{1}{t^{7 / 5}}>0 \quad(t \geq 2), \text { i.e., }-b / r \in \mathfrak{R}^{+}, \\
e_{-b / r}\left(t, t_{0}\right) & \geq 1-\int_{2}^{t} \frac{b(s)}{r(s)} \Delta s=1-\int_{2}^{t} s^{-\frac{12}{5}} \Delta s=1-\frac{t^{-\frac{7}{5}}-2^{-\frac{7}{5}}}{2^{-\frac{7}{5}}-1}=\frac{t^{-\frac{7}{5}}+1-2^{-\frac{2}{5}}}{1-2^{-\frac{7}{5}}} \\
> & 1-2^{-\frac{2}{5}}>\frac{1}{5}
\end{aligned}
$$

and so

$$
\begin{aligned}
\int_{t_{0}}^{t}\left[\frac{e_{-b / r}\left(s, t_{0}\right)}{r(s)}\right]^{1 / \lambda} \Delta s & \geq \int_{2}^{t}\left[\frac{1}{5 s^{2 / 5}}\right]^{7 / 3} \Delta s=\frac{1}{5^{7 / 3}} \int_{2}^{t} s^{-\frac{14}{15}} \Delta s \\
& =\frac{1}{5^{7 / 3}} \frac{t^{1 / 15}-2^{1 / 15}}{2^{1 / 15}-1} \rightarrow+\infty \quad(t \rightarrow+\infty) .
\end{aligned}
$$

Hence, conditions $\left(\mathrm{H}_{1}\right)-\left(\mathrm{H}_{5}\right)$ are clearly satisfied. Let $m=2$, in view of $\beta=1 / \sqrt{2}, L=6$, then we have

$$
\begin{aligned}
& \limsup _{t \rightarrow+\infty} \frac{1}{t^{m}} \int_{t_{0}}^{t}(t-s)^{m} \frac{L P(s)[1-\beta B(\delta(s))]^{\lambda}\left[h_{2}\left(\delta(s), t_{0}\right)\right]^{\lambda}}{[4 a(\delta(s)) \sigma(s)]^{\lambda}} \Delta s \\
& =\frac{6}{4^{3 / 7}} \limsup _{t \rightarrow+\infty} \frac{1}{t^{2}} \int_{2}^{t} \frac{(t-s)^{2}}{s} \Delta s \\
& =\frac{6}{4^{3 / 7}} \limsup _{t \rightarrow+\infty}\left\{\left[\log _{2}(t)-1\right]+\frac{1}{t^{2}}\left(\frac{t^{2}-2^{2}}{2^{2}-1}\right)-\frac{2}{t}(t-2)\right\}=+\infty, \\
& \limsup _{t \rightarrow+\infty} \frac{1}{t^{m}} \int_{t_{0}}^{t}(t-s)^{m} \frac{[b(s)]^{\lambda+1}}{[r(s)]^{\lambda}} \Delta s \\
& =\limsup _{t \rightarrow+\infty} \frac{1}{t^{2}} \int_{2}^{t} \frac{(t-s)^{2}}{s^{\frac{106}{35}}} \Delta s \leq \limsup _{t \rightarrow+\infty} \frac{1}{t^{2}} \int_{2}^{t} \frac{(t-s)^{2}}{s^{3}} \Delta s \\
& =\limsup _{t \rightarrow+\infty} \frac{1}{t^{2}}\left[\frac{t^{2}\left(t^{-2}-2^{-2}\right)}{2^{-2}-1}-\frac{2 t\left(t^{-1}-2^{-1}\right)}{2^{-1}-1}+\left[\log _{2}(t)-1\right]\right]<+\infty \text {. }
\end{aligned}
$$


This implies

$$
\begin{aligned}
& \limsup _{t \rightarrow+\infty} \frac{1}{t^{m}} \int_{t_{0}}^{t}(t-s)^{m}\left\{\frac{L P(s)[1-\beta B(\delta(s))]^{\lambda}\left[h_{2}\left(\delta(s), t_{0}\right)\right]^{\lambda}}{[4 \sigma(s) a(\delta(s))]^{\lambda}}-\frac{[b(s)]^{\lambda+1}}{(\lambda+1)^{\lambda+1}[r(s)]^{\lambda}}\right\} \Delta s \\
& \quad=+\infty
\end{aligned}
$$

and so conditions of Corollary 3.6 are satisfied as well. Altogether, by Corollary 3.6, we have that every solution of equation (4.2) is oscillatory or tends to zero as $t \rightarrow+\infty$. But the results in [9-16] are inapplicable for equation (4.2).

\section{Competing interests}

The author declares that he has no competing interests.

\section{Acknowledgements}

This work was supported by the Natural Science Foundation of Hunan Province (12JJ6006) and Hunan Province Science and Technology Project (2012FJ3107) and Scientific Research Fund of Hunan Provincial Education Department (09A082)

\section{Received: 3 February 2013 Accepted: 6 April 2013 Published: 19 June 2013}

\section{References}

1. Bohner, M, Peterson, A: Dynamic Equations on Time Scales: An Introduction with Applications. Birkhäuser, Boston (2001)

2. Agarwal, RP, Bohner, M, Li, WT: Nonoscillation and Oscillation: Theory for Functional Differential Equations. Dekker, New York (2004)

3. Sahiner, Y: Oscillation of second order delay differential equations on time scales. Nonlinear Anal. TMA 63, e1073-e1080 (2005)

4. Agarwal, RP, Bohner, M, Saker, SH: Oscillation of second order delay dynamic equations. Can. Appl. Math. Q. 13(1), 1-18 (2005)

5. Zhang, QX, Gao, L, Liu, SH: Oscillation criteria for second-order half-linear delay dynamic equations with damping on time scales (II). Sci. Sin. Math. 41(10), 885-896 (2011)

6. Zhang, $\mathrm{CH}$, Agarwal, RP, Bohner, M, et al.: New oscillation results for second-order neutral delay dynamic equations. Adv. Differ. Equ. 2012, 227 (2012)

7. Hassan, TS: Oscillation criteria for second-order nonlinear dynamic equations. Adv. Differ. Equ. 2012, 171 (2012)

8. Erbe, L, Peterson, A, Saker, SH: Hille and Nehari type criteria for third-order dynamic equations. J. Math. Anal. Appl. 329(1), 112-131 (2007)

9. Erbe, L, Hassan, TS, Peterson, A: Oscillation of third order nonlinear functional dynamic equations on time scales. Differ. Equ. Dyn. Syst. 18, 199-227 (2010)

10. Han, Z, Li, T, Sun, S, Cao, F: Oscillation criteria for third order nonlinear delay dynamic equations on time scales. Ann Pol. Math. 99(2), 143-156 (2010)

11. Han, Z, Li, T, Sun, S, Zhang, C: Oscillation behavior of third-order neutral Emden-Fowler delay dynamic equations on time scales. Adv. Differ. Equ. 2010, Article ID 586312 (2010)

12. Han, ZL, Li, TX, Sun, SR, et al.: Oscillation behavior of solutions of third-order nonlinear delay dynamic equations on time scales. Commun. Korean Math. Soc. 26(3), 499-513 (2011)

13. Hassan, TS: Oscillation of third order nonlinear delay dynamic equations on time scales. Math. Comput. Model. 49, 1573-1586 (2009)

14. Li, T, Han, Z, Sun, Y, Zhao, Y: Asymptotic behavior of solutions for third-order half-linear delay dynamic equations on time scales. J. Appl. Math. Comput. 36, 333-346 (2011)

15. Li, TX, Han, ZL, Zhang, CH, et al.: Oscillation criteria for third-order Emden-Fowler delay dynamic equations on time scales. Acta Math. Sci. 32(1), 222-232 (2012)

16. Yang, J, Sun, $\amalg, \mathrm{Xu}, \mathrm{LH}$ : Asymptotic and oscillatory behavior for third-order nonlinear neutral variable delay dynamic equations. Math. Appl. 23(3), 508-515 (2010)

17. Tuna, A, Kutukcu, S: Some integral inequalities on time scales. Appl. Math. Mech. 29(1), 23-29 (2008)

doi:10.1186/1687-1847-2013-178

Cite this article as: Yang: Oscillation criteria for certain third-order delay dynamic equations. Advances in Difference Equations 2013 2013:178. 